\title{
Analysis of genetic factors affecting egg production in Drosophila melanogaster
}

\author{
A. Dominguez and J. Rubio
}

Departamento de Genética, Universidad de Oviedo, 33071 Oviedo, España.

Genetic determinants of egg production were studied in two inbred lines of Drosophila melanogaster. Chromosome III was the only one responsible for differences in egg laying between them; its effect is completely dominant. By means of a marker stock, synthetic chromosomes were constructed consisting of known portions of the two third chromosomes but without carrying any marker genes themselves. Chromosome III was thus divided into seven regions. It was shown that genes with effect over the trait are concentrated on the left arm of the chromosome displaying complete dominance. A factor was located on the left end of this chromosomic arm having a major effect on the trait (33.10 \pm $4.96 \mathrm{eggs} / \mathrm{female} / \mathrm{day}$ ) by interaction with contiguous regions. A short segment including the located factor (or possibly the factor itself) acts as a dominance modifier and also as a segregation suppressor in contiguous segments.

\section{INTRODUCTION}

Several studies have attempted to ascribe differences in fecundity to specific chromosome segments in Drosophila melanogaster. Some of them (Gilbert, 1961; Chapco, 1968) were restricted to comparisons between segments homozygous and heterozygous for recessive markers. Consequently, any effects of dominant genes influencing egg production that were carried in the marker stocks could not be detected. Also, the possible pleiotropism of marker genes on egg laying could not be excluded. Other studies (Chapco, 1977; 1980) dealt with the effect of unmarked segments of chromosomes $\mathrm{X}$ and III, as large as a chromosomic arm. Therefore egg production genes could not be distinguished individually.

This paper goes one step further by using Thoday's method of location of polygenes (Thoday, 1961) in order to identify chromosomal factors displaying a major effect on egg production. This method was successfully applied to morphological traits in Drosophila melanogaster (see, for example, Thompson and Thoday, 1979) but has rarely been used to study traits directly related to fitness. Only Thomas-Orillard (1975) has attempted to locate genes affecting ovariole number and the results were inconclusive. This failure may be attributed to the complex genetical architecture of such traits as well as to the great environmental variance to which they are subjected.

We present an analysis of the distribution of genes causing a difference in egg production between two inbred lines from the same original population. The experimental conditions were greatly controlled to reduce environmental variance, essential in identifying the specific components of polygenic systems (Thoday, 1979).

\section{MATERIALS AND METHODS}

The lines used in these experiments were from a wild population of Drosophila melanogaster caught in Teverga (Spain), derived by inbreeding 60 generations of brother $\times$ sister mating. Two lines, named 1 and 4 , were chosen among seven inbred lines because of the larger difference in their egg production. Line 4 was completely dominant over line 1 (Domínguez, 1983).

All experiments were carried out in an incubator at $24 \pm 1^{\circ} \mathrm{C}$ with light-dark cycles of 12 hours.

Culture conditions and the method for determining egg production were the following: culture density was fixed by seeding 150 eggs per vial $(5.5 \mathrm{~cm} \times 15 \mathrm{~cm}$ ) with the medium (water, $1000 \mathrm{ml}$; agar, 12 g.; sugar, 100 g.; baker's yeast, 100 g. and 
propionic acid, $5 \mathrm{ml}$.) seeded with a drop of live baker's yeast suspension. Only the females emerging from these cultures during the first day were used in the oviposition experiments.

To determine egg production, females aged 8 hours or less and one-day-old virgin males were set up at three pairs per vial. The vials contained the culture medium dyed with $4 \mathrm{gr}$. of charcoal/1. and seeded with two drops of a 10 per cent suspension of live baker's yeast on the surface. Every day, flies were transferred to fresh medium and the eggs that had been laid were counted. Egg production was scored as the mean daily egg laying per female from the second to the seventh day of the female's age. This period included the same relative portions of the egg production curves of lines and hybrids, and this interval corresponds to the maximum daily yield (Domínguez, 1983).

All the experiments, except the comparison of the effect of chromosome $\mathrm{X}$, were repeated in several consecutive replicates with four egg laying vials per genotype and replicate. The Mean Square of interaction genotype $\times$ replicate was homogeneous between experiments $\left(\chi^{2}=2.76\right.$ in Bartlett's test of variance homogeneity) so it was pooled. This pooled MS interaction was significantly larger than the MS within genotype and replicate $(F=2 \cdot 47 ; p<0 \cdot 001)$. Therefore the pooled MS interaction genotype $\times$ replicate was used as MS error.

The effect of chromosome $\mathrm{X}$ on egg laying was compared in the heterozygous state since line 4 is completely dominant over line 1 . Homozygous $(1 / 1)$ or heterozygous $(1 / 4)$ females were bred from backcrossing reciprocal $F 1$ males to line 1 females. In this experiment, the egg laying scores were made for individual females kept with two males during the egg laying period. The density of cultures, although controlled, had not yet been fixed as described (this was done later on in order to reduce the environmental variance, Domínguez, 1983).

To study the effects of chromosomes II and III, we synthesised four true breeding combinations of these two chromosomes from lines 1 and 4 with their chromosome $\mathrm{X}$ from line 1 by Kearsey and Kojima's crossing scheme (1967). The inversion chromosomes used were Binscy for the $\mathrm{X}$ chromosome, SM5 Cy for chromosome II and TM3 Sb Ser for chromosome III. Each substitution line is referred to by three pair of numbers denoting the source of the X, 2nd and 3rd chromosome pairs. The egg laying of each of the nine chromosome II and III combinations was determined by two consecutive replicates.
To investigate the distribution of the genetic differences between the third chromosomes of lines 1 and 4 in respect to egg production, synthetic chromosomes consisting of two known portions of the two studied third chromosomes, were constructed by using the method of Breese and Mather $(1957 ; 1960)$. This method, more sophisticated than those usually employed in location studies, was also used by Thoday, Gibson and Spickett (1964).

For the construction of the synthetic chromosomes, the chromosome substitution lines 111111 and 111144 , as well as two stocks bearing the chromosomes rucuca and ruPrica, were used. The chromosome rucuca bears the recessive genes $r u, 0.0 ; h, 26 \cdot 5 ; t h, 43 \cdot 2 ; s t, 44 \cdot 0 ; c u, 50 \cdot 0$; $s r, 62 \cdot 0 ; e, 70.7$ and $c a, 100 \cdot 7$ (the markers $t h$ and $s t$ were treated as a single composite locus that is referred throughout as $s t$ ). The other stock bears the chromosomes ruPrica and TM3 SB Ser. The chromosome ruPrica has the same recessive markers as rucuca plus the dominant marker $\mathrm{Pr}$. The first and second chromosomes of these two stocks were replaced by those of line 1 by using the balancer chromosomes Binscy, SM5 Cy and TM3 Sb Ser. Thereafter, all the synthetic chromosomes were obtained in a line 1 background. For a complete description of the special chromosomes cited, see Lindsley and Grell (1968).

Six synthetic chromosomes of each single recombinant class were constructed so that chromosome III was treated as consisting of seven segments identified by letters $(\mathrm{A}, \mathrm{a}) ;(\mathrm{B}, \mathrm{b}) \ldots(\mathrm{G}, \mathrm{g})$ from left to right. Capital and small letters indicate segments belonging to the 4 and 1 chromosomes, respectively. The six synthetic chromosomes of the same class are numbered 1 to 6 . Chromosomes 1 to 3 and 4 to 6 were synthesized independently, but within each of these two groups they could proceed from the same or different "derived" chromosomes (see Thoday, Gibson and Spickett, 1964).

Females of different genotypes for the segments in question can be bred by crossing synthetic chromosome (i)/ TM3 males to synthetic chromosome (j)/ TM3 females.

Finally, synthetic chromosomes, recombined between markers $r u$ and $h$, were constructed to assay the number of factors (see Thoday, 1961) responsible for the fecundity difference between chromosomes aBCDEFG (low phenotype) and 4 (=ABCDEFG, high phenotype) (see results). These synthetic chromosomes were constructed from chromosome $n^{0} 1$ of class aBCDEFG, which brings the right segment, and chromosome 4 , which 
brings the left segment. Twelve, in some cases less due to failure, synthetic chromosomes were obtained from each of seven A $h$ st $c u$ sr e ca derived chromosomes, each one combined with four $r$ BCDEFG derived chromosomes. Homozygous mean egg laying given by these synthetic chromosomes was determined in a replicate of egg counts consisting of two vials per synthetic chromosome. Chromosomes which could not be clearly assigned to a phenotypic class in this first replicate were retested.

\section{RESULTS}

Mean egg laying of females homozygous for chromosome $X$ of line $1(67 \cdot 31 \pm 2 \cdot 34)$ and heterozygous $(71 \cdot 33 \pm 2 \cdot 51)$ did not differ $(t=1 \cdot 15$; $p>0 \cdot 05)$. Therefore the difference between lines 1 and 4 is not caused by the $\mathrm{X}$ chromosome.

The analysis of variance on chromosome II and chromosome III effects (table 1) reveals that chromosome III is the only one responsible for the difference between lines 1 and 4 and its effect is almost completely dominant. Since culture density in the test on chromosome $\mathrm{X}$ effect was not controlled in the following experiments, it seems worth noting here that mean egg laying of females 114444 did not differ from that of line 4 females $(87 \cdot 87 \pm 3 \cdot 33 \mathrm{eggs} /$ female/day $)$ which were scored simultaneously as control.

In the analysis on chromosome III, some synthetic chromosomes were found to be lethal or to

Table 1 Mean egg laying of chromosome II and chromosome III substitution lines (a) and analysis of variance (b) a) Mean egg laying

\begin{tabular}{ccccc}
\hline & \multicolumn{4}{c}{ Chrom. III } \\
\cline { 2 - 5 } & 11 & 14 & 44 & $\overline{\mathrm{X}}$ \\
\hline Chrom. II & & & & \\
11 & $67 \cdot 99$ & $90 \cdot 85$ & $90 \cdot 95$ & $83 \cdot 26$ \\
14 & $69 \cdot 49$ & $96 \cdot 04$ & $93 \cdot 61$ & $86 \cdot 38$ \\
44 & $62 \cdot 78$ & $91 \cdot 51$ & $85 \cdot 25$ & $79 \cdot 85$ \\
$\overline{\mathrm{X}}$ & $66 \cdot 75$ & $92 \cdot 80$ & $89 \cdot 94$ & \\
\hline
\end{tabular}

b) Analysis of variance

\begin{tabular}{|c|c|c|c|}
\hline Source & d.f. & M.S. & $F$ \\
\hline Chromosome II & 2 & 255.86 & $2 \cdot 88$ \\
\hline Chromosome III & 2 & $4898 \cdot 55$ & $55 \cdot 10^{*}$ \\
\hline Chrom. II $\times$ Chrom. III & 4 & $26 \cdot 43$ & $<1$ \\
\hline $\begin{array}{l}\text { Error (pooled genotype } \\
\times \text { replicate interaction) }\end{array}$ & 142 & $88 \cdot 91$ & \\
\hline
\end{tabular}

* $p<0.001$ cause sterility in the homozygous state or when heterozygous with a chromosome TM3. So precaution is a must when interpreting egg laying means given by such chromosomes, encircled in the graphics.

Heterozygous mean egg laying given by synthetic chromosome $n^{\circ} 1$ of each class ( 4 replicates) are presented in fig. 1. The most important effects are displayed by the $(B, b)$ segment (effect associated with the $h$ marker), when increasing the length of chromosome segments from line 4 replacing those from line 1 from left to right. Yet segments $(A, a)$ and $(C, c)$ have a larger effect (effects associated with the markers $r u$ and st respectively) when segments from line 4 are replaced by those from line 1 from left to right.

Egg laying given by all the chromosomes from the classes recombined between these and contiguous markers was determined afterwards. In this way, it could be possible to know at which side of the markers the relevant factors are (Thoday, 1961). Analysis of variance of chromosomes within each class (table 2) shows that there are some segregating factors affecting egg laying within classes ABcdefg, abCDEFG and abcDEFG.

Chromosome means are presented as deviations from homozygous $1 / 1$ control mean so as to compare experiments that were not performed simultaneously (fig. 2). Segregation in class ABcdefg must be due to chromosome $n^{\circ} 5$, which was later shown to produce homozygous sterility, and therefore is not taken into account. There is no easy interpretation for segregation in classes abCDEFG and abcDEFG (between the markers $h-s t$ and $s t-c u$ respectively) for two reasons. First, chromosomes of class aBCDEFG, namely with a short segment from line 1 chromosome, already reach egg laying means similar to that of control $1 / 1$. Second, means of egg laying reaching the lowest mean of class abcDEFG are not found in successive classes with larger segments from line 1 chromosome (see fig. 1). Segregation in classes abCDEFG and abcDEFG could be caused by the short piece of rucuca chromosome which may or may not be interposed between 4 and 1 segments, forming each synthetic chromosome. Other explanations imply complex genetic interactions among genes from the two lines in the different segments.

Segment $(A, a)$ is the only one with a clear effect on egg production; the substitution of $\mathbf{A}$ with a, the rest of the chromosome being from line 4 , has an effect nearly equal to the difference between the lines, while the effect of this segment when the rest of chromosome is from line 1 is small. All the factors responsible for the difference between lines 


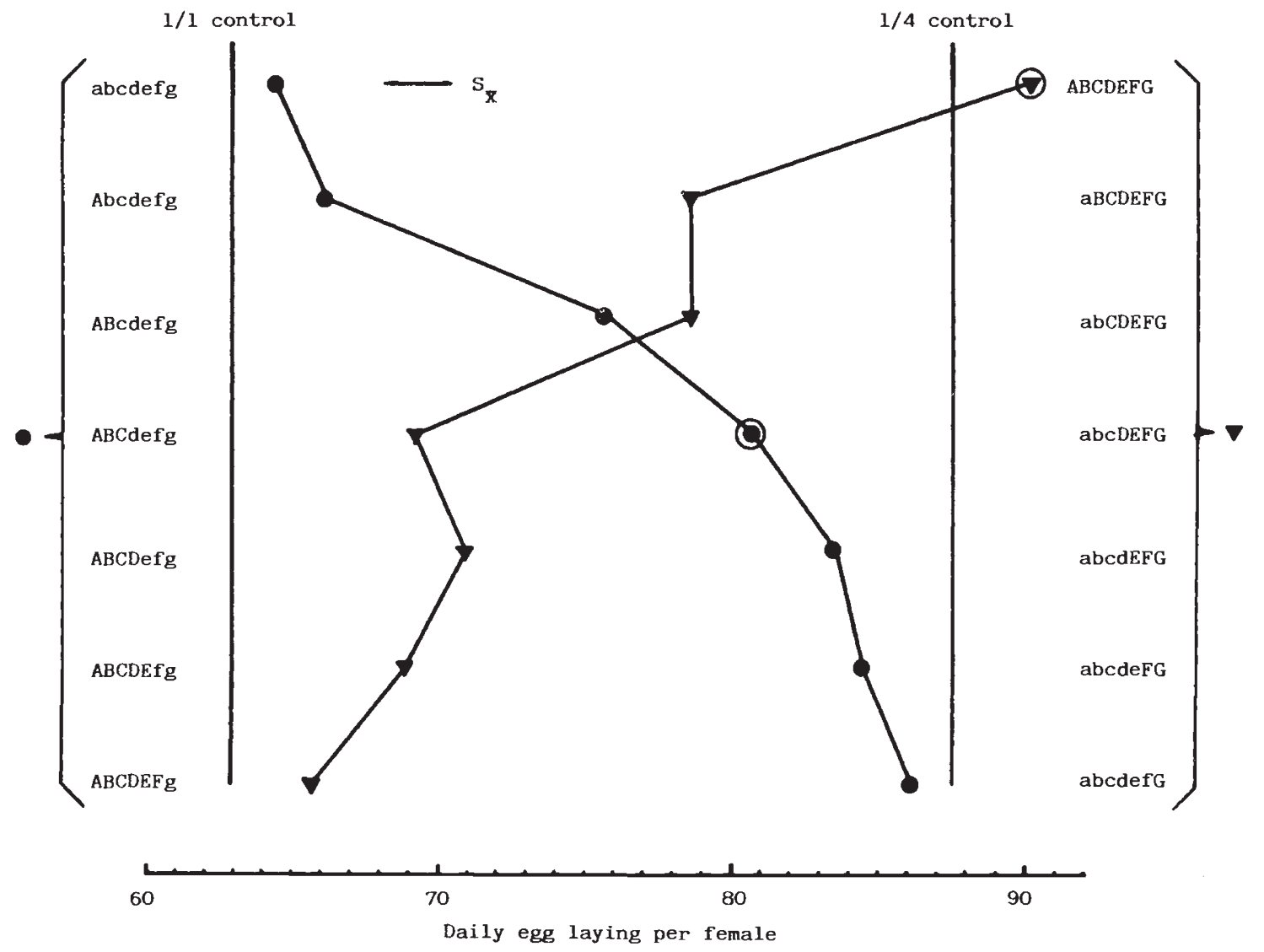

Figure 1 Mean egg laying given by a synthetic chromosome from each class when heterozygous with a third chromosome from line 1. Chromosomes that were later shown to be homozygous sterile or lethal are sorrounded by a circle.

1 and 4 are located on the left arm of chromosome III $((A, a) ;(B, b)$ and $(C, c)$ segments $)$, since the $\mathrm{ABCdefg}$ chromosomes already reach the egg laying rate of control $1 / 4$.

Homozygous and heterozygous mean egg laying given by one chromosome from each of the

Table 2 Analysis of variance of chromosomes within each of classes which recombined at the left arm of chromosome III when heterozygous with a line 1 chromosome

\begin{tabular}{lrrl}
\hline Source & d.f. & M.S. & F \\
\hline Between chromosomes within class: & & & \\
$\quad$ Abcdefg (2 replicates) & 5 & 13.02 & $<1$ \\
aBCDEFG (2 replicates) & 5 & $66 \cdot 23$ & $<1$ \\
ABcdefg (4 replicates) & 5 & $453 \cdot 35$ & $5 \cdot 10^{* *}$ \\
abCDEFG (4 replicates) & 5 & $389 \cdot 63$ & $4 \cdot 38^{* *}$ \\
ABCdefg (2 replicates) & 3 & 108.62 & $1 \cdot 22$ \\
$\quad$ abcDEFG (2 replicates) & 4 & $251 \cdot 64$ & $2 \cdot 83^{*}$ \\
Error (pooled genotype & & & \\
$\quad \times$ replicate interaction) & 142 & 88.91 & \\
\hline
\end{tabular}

${ }^{*} \mathrm{p}<0.05 ;{ }^{* *} \mathrm{p}<0.001$. classes recombined on the left arm of chromosome III (chromosome $n^{\circ} 1$ except for class ABCdefg from which the tested chromosome was $n^{\circ} 4$ because chromosomes $n^{\circ} 1, n^{\circ} 2$ and $n^{\circ} 3$ were homozygous sterile or lethal) is presented in fig. 3 (3 replicates). Every chromosome bearing the A segment displays complete dominance, while synthetic chromosomes with the a segment show overdominance. The effect of substituting $A$ with a in an otherwise chromosome 4 is much more important than the substitution of this segment in an otherwise chromosome 1 . This fact, also seen in fig. 2 , is more obvious when chromosomes are homozygous. Therefore the effect of the A segment is due to interaction with other genes from chromosome 4 which must be in segments $B$ and $C$ since the $\mathrm{ABCdefg}$ chromosome is equivalent to chromosome 4 .

The chromosome abCDEFG gave a larger homozygous mean egg laying than the chromosome aBCDEFG, that is, the presence of the $b$ segment (from line 1) instead of segment B (from 


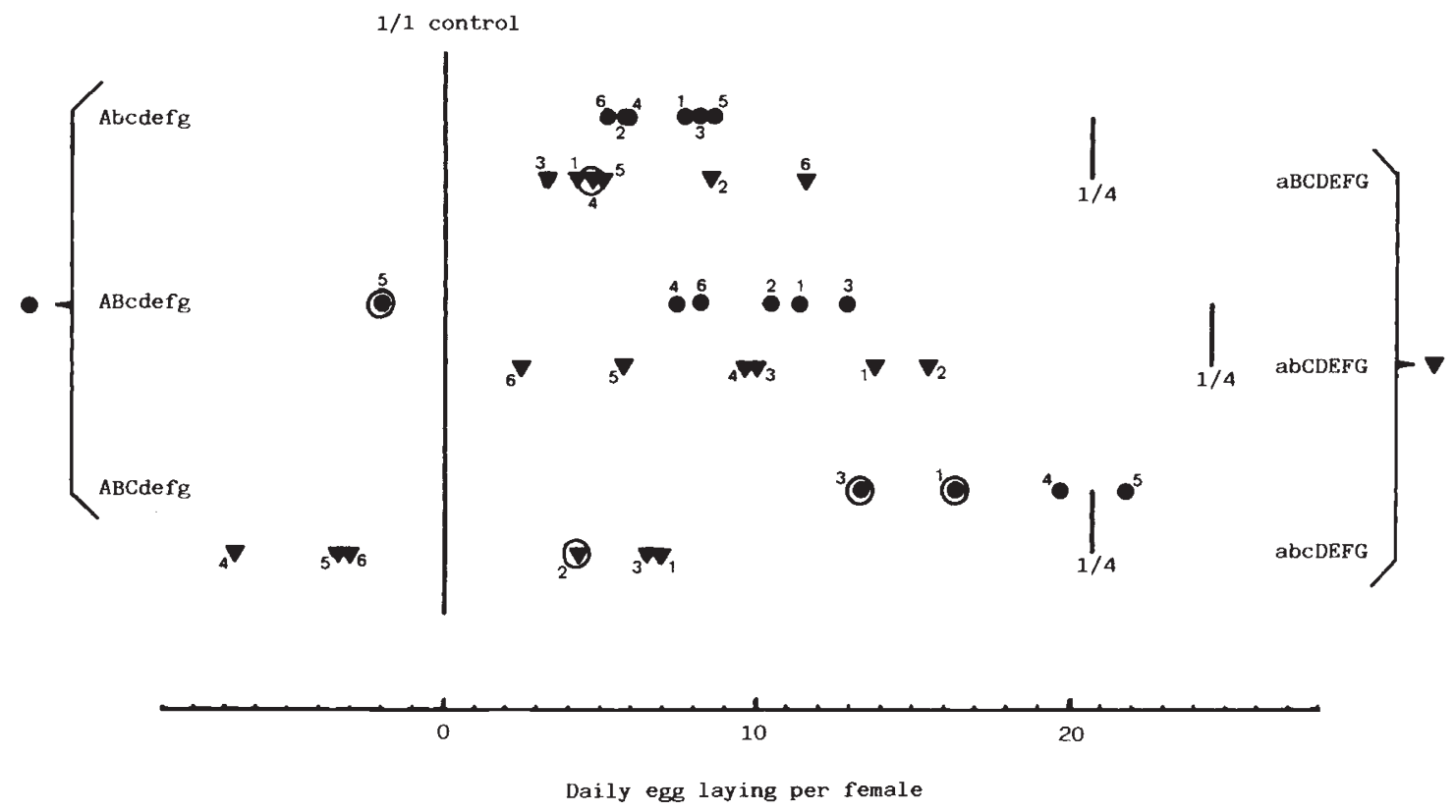

Figure 2 Mean egg laying given by several synthetic chromosomes of classes which recombined at the left arm of chromosome III when heterozygous with a third chromosome from line 1 . Means are expressed as differences from $1 / 1$ control.

line 4), clearly increases egg laying, contrary to the trend observed when comparing Abcdefg and ABcdefg chromosomes; this increase, however, is not maintained in chromosome abcDEFG, when segment $c$, from line 1 , is added. The same trend can be observed in the heterozygous condition. This effect of segment $(B, b)$ is caused by genes which are segregating in class abCDEFG. The heterozygous segregation of such genes has been shown previously (table 1 and fig. 2), and their homozygous effect is more important (fig. $4 ; p<$ $0 \cdot 001$ ).

There were not significant differences between the six chromosomes within class abCDEFG, nor any between the two more extreme chromosomes within class abcDEFG when heterozygous with an Abcdefg chromosome (table 3). Furthermore, chromosomes from class abCDEFG (fig. 5) gave

Table 3 Analysis of variance of chromosomes within each of classes abCDEFG and abcDEFG when heterozygous with an Abcdefg chromosome

\begin{tabular}{lrrl}
\hline Source & d.f. & M.S. & F \\
\hline Between chromosomes within class: & & & \\
$\quad$ abCDEFG & 5 & 49.05 & $<1$ \\
$\quad$ abcDEFG & 1 & 2.08 & $<1$ \\
Error (pooled genotype & & & \\
$\quad \times$ replicate interaction) & 142 & 88.91 & \\
\hline
\end{tabular}

a lower mean than chromosome aBCDEFG (although the difference is not significant), contrary to the trend observed when these chromosomes were heterozygous with a chromosome from line 1 (see fig. 1, 2 and 3). Therefore, the effect of segregating genes in classes abCDEFG and abcDEFG is suppressed by the A segment.

Finally, in order to ascertain the number of factors responsible for the effect of the $(A, a)$ segment, 79 synthetic chromosomes were constructed from chromosomes aBCDEFG (low phenotype) which brings the right segment and 4 (= ABCDEFG; high phenotype) which brings the left segment, the junction lying between the loci of the markers $r u$ and $h$. Thirty-six such chromosomes were abnormal for viability or fertility, so egg laying was scored only for the remaining 43 chromosomes. All these chromosomes give egg laying means close to that of the chromosome 4 control and well separated from the aBCDEFG control. Retesting of those synthetic chromosomes with more extreme means confirms this fact (fig. 6). Therefore, the major effect of the $(A, a)$ segment must be due to a factor which maps near locus $\mathrm{ru}$, since among the 43 synthetic chromosomes recombined between the $r u$ and $h$ markers no recombination was observed between the factor under study and the locus $r u$. Therefore, the maximum distance between these two loci with 99 per cent confidence must be of $2.65 \mathrm{cM}$. There may also be some other 


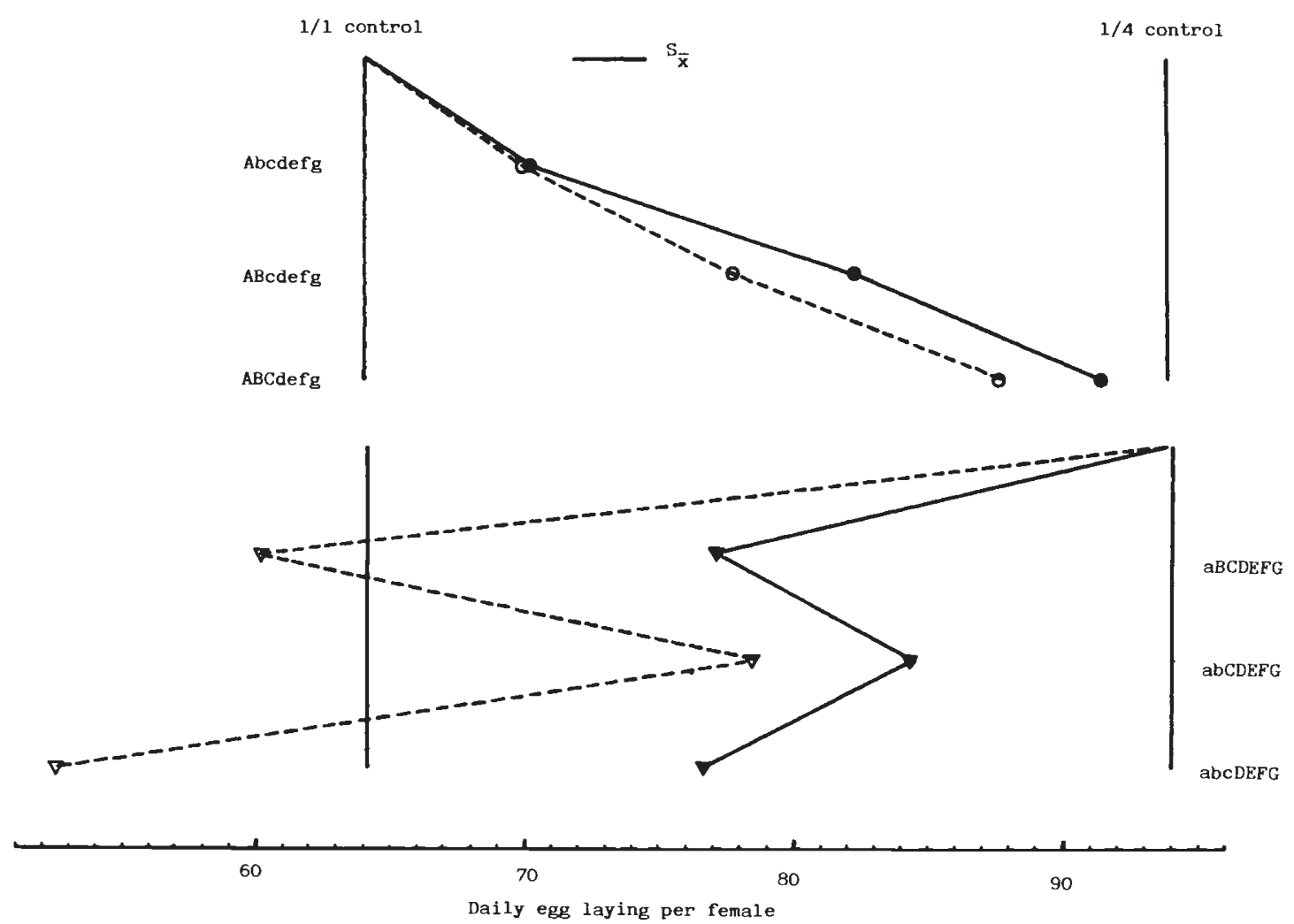

Figure 3 Homozygous - - - and heterozygous __ mean egg laying given by a synthetic chromosome from each of classes which recombined at the left arm of chromosome III.

factors in this region with a minor effect over the trait since there were significant $(p<0 \cdot 05)$ differences among synthetic chromosomes. By comparing the mean egg laying associated with the 43 new synthetic chromosomes and the egg laying mean of chromosome aBCDEFG, the main factor in segment $(A, a)$ has a homozygous mean effect of $33 \cdot 10 \pm 4 \cdot 96 \mathrm{eggs} / \mathrm{female} / \mathrm{day}$. As was shown, this effect is due to interaction with other factors in segments $(B, b)$ and $(C, c)$.

\section{DISCUSSION}

An important problem when studying egg production and fertility traits in general is their great sensitivity to environmental variation. This sensitivity makes it difficult to obtain repeatable results since it frequently introduces genotype $\mathrm{x}$ environment interaction which obscures the genetic differences under study (Chapco, 1968; 1977; FitzEarle, 1972). In this study the genotype $\mathrm{x}$ replicate interaction was greatly reduced by the great control over the experimental conditions. Although sig- nificant, it was small enough not to conceal the genetic differences studied.

In contrast to Gowen (1952) and Robertson and Reeve (1955), the present study shows that there is no relation between the number of heterozygous chromosomes and egg production. Interchromosomal interactions (Robertson and Reeve, 1955; Keller and Mitchell, 1964; Fitz-Earle, 1972) were not found.

Although there may be some association between egg production and the amount of heterozygosity within chromosome III (fig. 1), this relation is in no case linear and there are genotypic combinations which are largely heterozygotic and which show some of the shortest egg laying means (see, for example, chromosome abcDEFG $n^{\circ} 4$ or chromosome aBCDEFG $n^{\circ} 3$ in fig. 2). Similarly, Chapco (1977) showed that there was a limited correspondence between egg production and heterozygous length within the $\mathrm{X}$ chromosome, and the same was shown by Breese and Mather (1960) in relation to relative viability and the number of heterozygous segments within the third chromosome. 


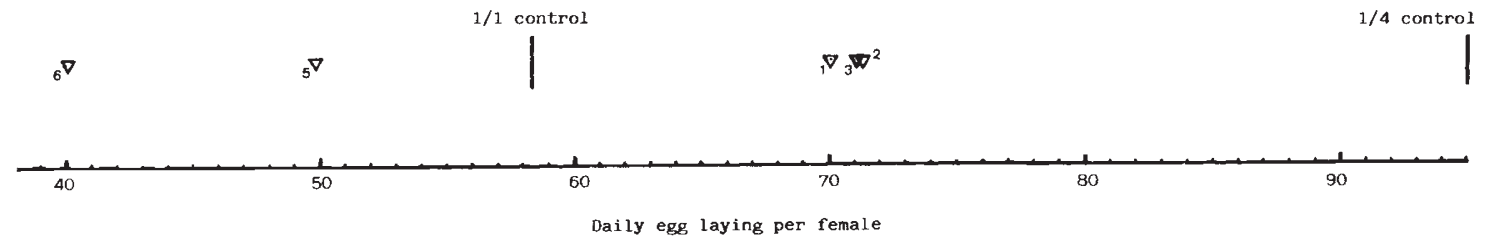

Figure 4 Homozygous mean egg laying given by five chromosomes of class abCDEFG.

Directional dominance (or overdominance) and intersegmental interactions were present (fig. 3). These features were to be expected since they are fairly common when studying the genetic architecture of fitness traits that are supposed to have been exposed to directional selection in the past (Breese and Mather, 1960; Kearsey and Kojima, 1967).

Segment $(\mathrm{A}, \mathrm{a})$ acts as a dominance modifier of contiguous segments (fig. 3). This effect may be explained if segment A suppresses or reduces the effect of homozygous low fecundity genes in other segments. This explanation is based on the fact that the presence of the A segment suppresses the segregation in synthetic chromosome classes abCDEFG and abcDEFG. That the degree of dominance of a segment depends on the genetic constitution at other segments has already been shown by Chapco $(1977 ; 1980)$ among long chromosome segments, and among entire chromosomes for fecundity by Keller and Mitchell (1964). This feature was also found by Morton et al. (1968) and Mukai (1969), who showed that the degree of dominance of several chromosomes with regard to viability depends on background heterozygosity. But, to our knowledge, it has not been previously

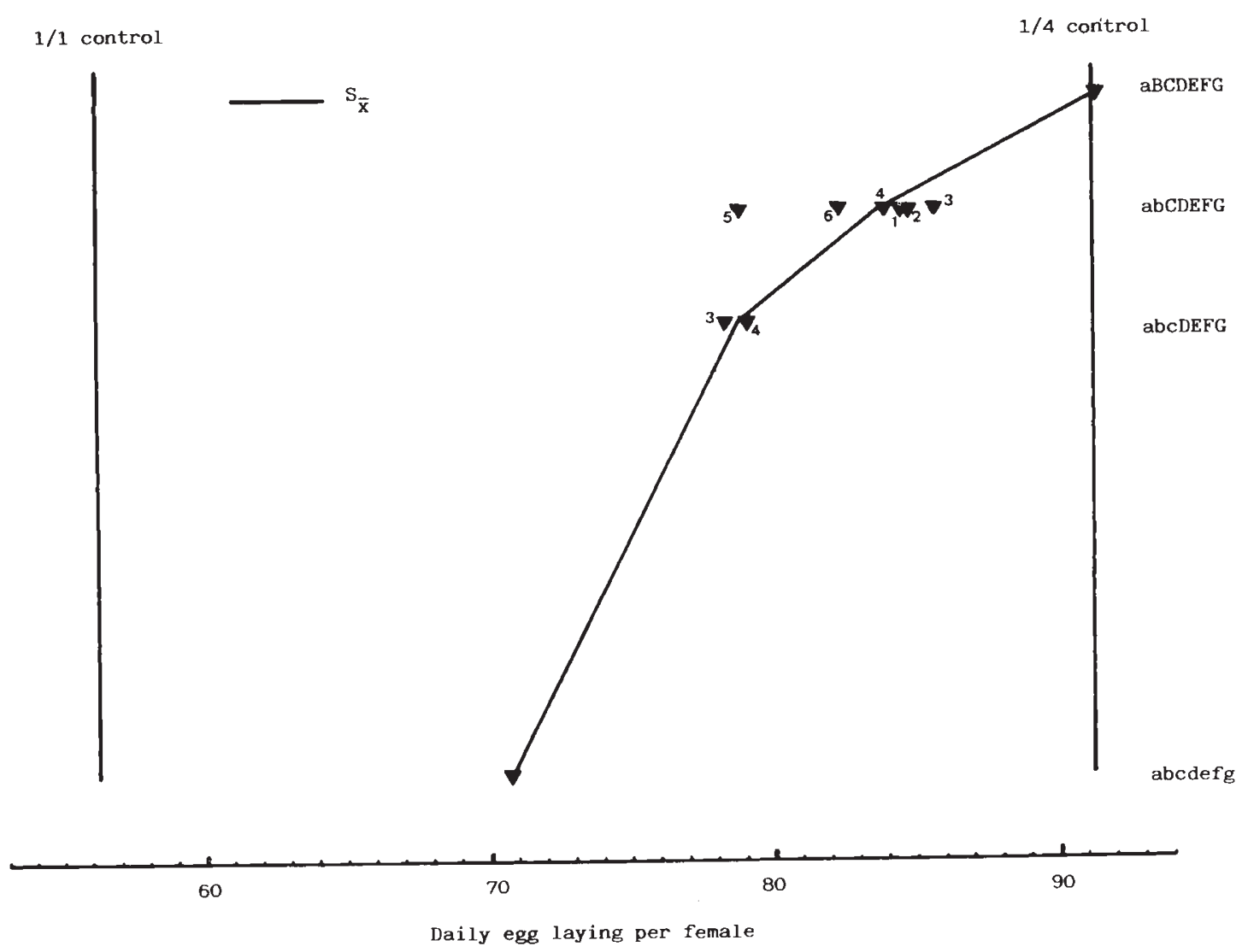

Figure 5 Mean egg laying given by synthetic chromosomes of classes aBCDEFG, abCDEFG, abcDEFG and abcdefg when heterozygous with an Abcdefg chromosome. 


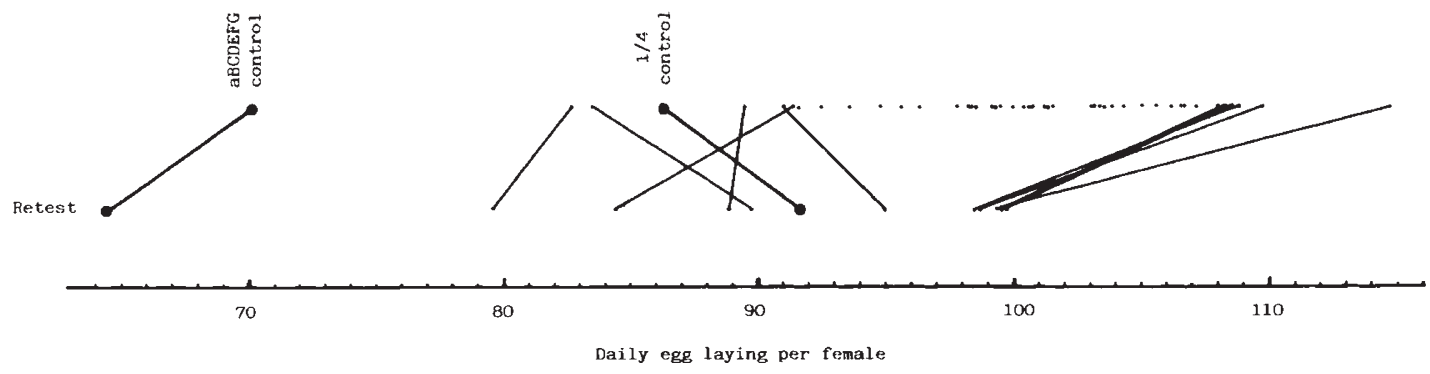

Figure 6 Distribution of homozygous mean egg laying given by synthetic chromosomes recombined between $r u$ and $h$ markers (see the text). Means were estimated from a replicate and retest of chromosomes with more extreme means was made.

reported for a short segment, or possibly a single factor, which suppresses or reduces variation caused by segregation at other segments.

Finding lethal chromosomes among synthetic ones is not new in these kind of studies. Breese and Mather (1960) found such lethals among third synthetic chromosomes and suggested that they were synthetic or recombinational lethals. Gibson and Thoday (1962) also showed the occurrence of a lethal chromosome II when analysing a selected line and demonstrated that it was produced by recombination. The lethals we found could be synthetic, although, the possibility of them being lethals carried by the original lines cannot be discarded. Another possibility to take into account is lethal induction by hybrid dysgenesis (Kidwell, Kidwell and Sved, 1977; Bregliano et al., 1980) since we were dealing with matings among individuals from different lines to make chromosome substitution lines and synthetic chromosomes.

Since segregation was found among chromosomes within class, even when crossing-over has been restricted to a narrow region in map units (44.0-50.0 for the abcDEFG class), care must be taken in interpreting the results for chromosome segment effect based on just one synthetic chromosome per class.

Distribution and effect of fecundity genes in the analysis presented show some different features than other intrachromosomal analysis of fitness traits (Chapco, 1968; 1977; 1979; 1980; Breese and Mather, 1960; Gilbert, 1961; Thomas-Orillard, 1975). Fecundity genes differentiating the studied lines are concentrated on a chromosomic arm which has a completely dominant effect. Furthermore, we could identify in this chromosomic arm a factor with a major effect over the trait. The assumption of Chapco (1977) that "egg production genes are probably of small effect and hence cannot be individually distinguished" may be true when there are several environmental and genetic sour- ces of variation which can conceal differences under study, but individual factors with a major effect over the trait can be identified when interferences produced by other sources of variation are reduced.

Acknowledgement We thank Dr J. M. Thoday for his helpful comments on the choice of an adequate method for the third chromosome analysis.

\section{REFERENCES}

BREESE, E. L. AND MATHER, K. 1957. The organisation of polygenic activity within a chromosome in Drosophila. I. Hair characters. Heredity, 11, 373-395.

BREESE, E. L. AND MATHER, K. 1960. The organisation of polygenic activity within a chromosome in Drosophila. II. Viability. Heredity, 14, 375-399.

BRegliano, J. C., PICARD, G., BUCheton, A., PELISSON, A., LAVIGE, J. M. AND L'HERITIER, P. 1980. Hybrid dysgenesis in Drosophila melanogaster. Science, 207, 606-611.

CHAPCO, w. 1968. Correlations between egg production and chromosomal regions in Drosophila melanogaster. Can. J. Genet. Cytol., 10, 951-960.

CHAPCO, w. 1977. Correlations between chromosome segments and fitness in Drosophila melanogaster. I. The X chromosome and egg production. Genetics, 85, 721-732.

CHAPCO, W. 1979. Correlations between chromosome segments and fitness in Drosophila melanogaster. II. The X chromosome and egg vibvility. Genetics, 92, 595-601.

CHAPCO, w. 1980. Correlations between chromosome segments and fitness in Drosophila melanogaster. IV. Fecundity, viability and the third chromosome. Can. J. Genet. Cytol, 22, 405-410.

DOMINGuez, A. 1983. Análisis de la arquitectura genética de un carácter de eficacia biológica: La puesta de huevos en Drosophila melanogaster. Tesis Doctoral. Universidad de Oviedo.

FITZ-EARLE, M. 1972. Quantitative genetics of fertility. IV. Chromosomes affecting egg production in Drosophila melanogaster. Can. J. Genet. Cytol., 14, 147-156.

GIBSON, J. B. AND THODAY, J. M. 1962. Effects of disruptive selection. VI. A second chromosome polymorphism. Heredity, 17, 1-26.

GILBERT, N. 1961. Quantitative inheritance in Drosophila. $J$. Genet., 57, 77-83. 
KEARSEY, M. J. AND KOJIMA, K. 1967. The genetic architecture of body weight and egg hatchability in Drosophila melanogaster. Genetics, 56, 23-37.

KELLER, E. C. JR. AND MITCHELL, D. F. 1964. Interchromosomal genotypic interactions in Drosophila. II. An analysis of viability characters. Genetics, 49, 293-307.

KIDWELL, M. G., KIDWELL, J. F. AND SVED, J. A. 1977. Hybrid dysgenesis in Drosophila melanogaster: a syndrome of aberrant traits including mutation, sterility and male recombination. Genetics, 86, 813-833.

LINDSLEY, D. L. AND GRELL, E. H. 1968. Genetic Variations of Drosophila melanogaster. Carnegie Inst. Wash. Publ. 627.

MORTON, N. E., CHUNG, C. S., FRIEDMAN, L. D., MORTON, N. T., MIKI, C. AND YEE, S. 1968. Relation between homozygous viability and average dominance in Drosophila melanogaster. Genetics, 60, 601-614.

MUKAI, T. 1969. The genetic structure of natural populations of Drosophila melanogaster. VII. Synergistic interaction of spontaneous mutant polygenes controlling viability. Genetics, 61, 749-761.
ROBERTSON, F. W. AND REEVE, E. C. R. 1955. Studies in quantitative inheritance. VIII. Further analysis of heterosis in crosses between inbred lines of Drosophila melanogaster. Z. Indukt. Abst. Vererb., 86, 439-458.

THODAY, J. M. 1961. Location of polygenes. Nature, 191, 368370.

THODAY, J. M. 1979. Polygene mapping: uses and limitations. In Quantitative Genetic Variation. Thompson, J. N. Jr. and Thoday, J. M. (eds), Academic Press.

THODAY, J. M., GIBSON, J. B. AND SPICKETT, S. G. 1964. Regular responses to selection. 2. Recombination and accelerated response. Genet. Res., 5, 1-19.

THOMAS-ORILLARD, M. 1975. Tentative delocalisation des génes qui président au déterminisme du nombre d'ovarioles chez la drosophila. Archiv. fur Genetick, 48, 116-127.

THOMPSON, J. N. (JR.) AND THODAY, J. M. (eds). 1979. Quantitative Genetic Variation. Academic Press. 